\title{
Equilibria and Price of Anarchy in Parallel Relay Networks with Node Pricing
}

\author{
Yufang Xi and Edmund M. Yeh \\ Department of Electrical Engineering \\ Yale University \\ New Haven, CT 06520, USA \\ Email: \{yufang.xi, edmund.yeh\}@yale.edu
}

\begin{abstract}
We study pricing games in single-layer relay networks where the source routes traffic selfishly according to the strategic bids made by relays. Each relay's bid includes a charging function and a proposed traffic share. Relays aim to maximize their individual profit from forwarding traffic. We show that the socially optimal traffic allocation can always be induced by an equilibrium where no relay can increase its profit by unilaterally changing its bids. Inefficient equilibria arise due to the monopolistic pricing power of a superior relay. This lead to a finite price of anarchy if marginal cost functions are concave, and an unbounded price of anarchy when the marginal cost functions are convex.
\end{abstract}

\section{INTRODUCTION}

It has been widely recognized that cooperation in networks formed by autonomous and selfish nodes cannot be achieved unless sufficient incentives are provided to the nodes. Such incentives normally take the form of payments or rewards to the nodes if they help forward other nodes' traffic [1]-[4]. A node is usually willing to participate in routing only if it can charge more than the cost of servicing the transit traffic. While a selfish node always prices its service with the ultimate aim of maximizing its profit, it has to do so strategically since the customers it courts may potentially buy services from other nodes. Thus, there exists a trade-off in each node's pricing decision. That is, higher charges potentially yield larger profit margins but risk losing market share to its competitors.

In this work, we study games that arise from the selfish and strategic pricing behavior of relay nodes in single-layer relay networks with one source and one destination. This type of games will be referred to as oligopolies. A node is selfish in the sense that maximizing its own profit is its sole objective. Being strategic means that a node is able to optimally design its bids based on the its competitors' strategies and its customer's response.

In an oligopoly pricing game, the source needs to forward its traffic to a set of relays, who in turn send the traffic to the destination. As a service provider to the source, each relay announces (1) a charging function which specifies the payments it demands from the source depending on the amount of traffic allocated by the source and (2) a proposed traffic share which helps the source decide on a unique traffic

\footnotetext{
${ }^{1}$ This research is supported in part by NSF grant CNS-0626882 and AFOSR grant FA9550-06-1-0135.
}

allocation in case it is indifferent among multiple (optimal) allocations. ${ }^{2}$ Given relays' bids, the source allocates its total traffic in a way that minimizes the sum of its own transmission costs and the payments made to relays. The games considered here can exist in both wireline and wireless networks, where communication consumes resources and nodes are often selfish agents such as ISPs. Despite the simple topology, the oligopoly games studied in this paper aptly model the local competitions that may exist in networks with more sophisticated topologies. In this respect, the oligopoly games serve as building blocks for games with multiple layers of relays and more general multi-hop network structure.

Pricing schemes were introduced into network resource allocation problems first as a means of decomposing a global optimization into sub-problems solved by individual agents [5]. In addition to being a facilitating device, pricing serves as an essential mechanism for inducing social optimum when users (source nodes) selfishly choose their routes [6]. It is well known that without appropriate pricing, e.g. marginal cost pricing, selfish routing inevitably results in loss of efficiency, which in general can be arbitrarily large [7], [8].

When service providers are also mindful of their own interest, they will use pricing to their own advantage rather than to heed any social mission. With both users and service providers behaving selfishly, the network increasingly approximates a free market, where prices assume a variety of functions and lead to direct or indirect competition among service providers. For example, pricing network services according to their quality helps to match each type of service with the customers that value it the most [9], [10]. By modelling the interaction between the service provider and the users as a Stackelberg game, [11] shows that when the service provider always adopts the profit-maximizing price, its revenue per unit bandwidth and the net utility of each user both improve with the number of users. When multiple service providers are present in a network, price competition inevitably ensues [12]-[14]. It is demonstrated in [12], [13] that cooperation in pricing is in the best interest of service providers who jointly serve the same customers. The consequence of non-cooperation is analyzed in [14], which shows that price competition in parallel-serial networks can cause arbitrarily large efficiency loss.

\footnotetext{
${ }^{2}$ The necessity of the second part is explained in detail later.
} 
A distinctive feature of the games studied in this work is that the bid from each relay to the source includes a (possibly nonlinear) charging function, which specifies the payment contingent on the amount of service provided. Previous work on pricing games almost exclusively assume a constant unit price (linear charging function) from every service provider, which is reasonable if the users being charged are nonatomic [6], [7], [14], [15], i.e., each user has infinitesimal traffic. Our work is among the relative few that investigate the behavior of atomic users [8] and the relays servicing them. Since the user now has non-negligible traffic that can be arbitrarily split and sent to multiple relays, it is necessary to consider the use of nonlinear charging functions to specify service fees. It turns out that the generalization from constant unit prices to nonlinear charging functions allows for a much richer set of possibilities in pricing games. Even in economics literature, the issue of nonlinear pricing is relatively new and proves to be quite challenging [16].

We show that an oligopoly pricing game always has Nash equilibria where no relay can increase its profit by unilaterally changing its bids. The traffic allocation at an equilibrium, however, may or may not be socially optimal. We show that the game always has efficient equilibria. In other words, the price of stability, defined as the ratio of the minimum cost at an equilibrium to the cost at the social optimum, is equal to one. Typically, inefficient equilibria also exist and they are always monopolistic, i.e., a dominant relay carries all the flow from the source. The ratio of the maximum equilibrium cost to the cost at the optimum, or the price of anarchy, is upper bounded by the number of relays if marginal cost functions are concave. In this case, the worst inefficient equilibria arise from symmetric oligopolies with linear marginal cost functions. When marginal cost functions are convex, however, the price of anarchy can be arbitrarily large.

\section{Network Model And Problem Formulation}

\section{A. Network Traffic and Multi-hop Routing}

We consider a relay network represented by a directed graph $\mathcal{G}=(\mathcal{N}, \mathcal{E})$ where the node set $\mathcal{N}$ consists of one source $s$, one destination $w$, and a single layer of parallel relays $\mathcal{I}$ which can be used to forward traffic from $s$ to $w$. A typical topology is illustrated in Figure 1. Here, $N$ relays each have a direct link

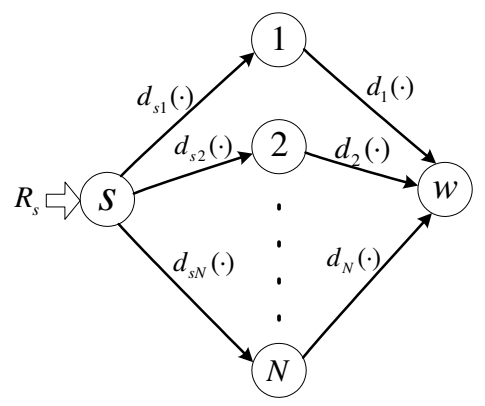

Fig. 1. Oligopoly with $\mathrm{N}$ relays. from $s$ and a direct link to $w$. We will explain other notations in Figure 1 shortly. To make the problem interesting, we assume that there is no direct link between $s$ and $w$ and there are at least two relays. The source $s$ needs to send traffic of a fixed rate $R_{s}$, which is to be allocated to the relays. Pricing games arising from such topologies will be referred to as oligopolies.

Denote by $f_{i}$ the rate of flow sent through relay $i$. It is implicitly assumed throughout the paper that $f_{i} \geq 0$. A traffic allocation vector $\boldsymbol{f} \triangleq\left(f_{i}\right)_{i \in \mathcal{I}}$ is said to be feasible if $\sum_{i \in \mathcal{I}} f_{i}=R_{s}$.

\section{B. Link Cost and Charging Functions}

Each link $(i, j) \in \mathcal{E}$ has a strictly increasing and strictly convex cost function $D_{i j}\left(f_{i j}\right)$, where $f_{i j}$ denotes the flow rate on link $(i, j)$. Without loss of generality, we assume that $D_{i j}(0)=0 .{ }^{3}$ Due to the single-layer topology, $f_{s i}=f_{i w}=f_{i}$. The cost $D_{i j}\left(f_{i j}\right)$ can, for example, represent the queuing delay incurred on $(i, j)$ with arrival rate $f_{i j}$, e.g. the average occupancy function $f_{i j} /\left(c_{i j}-f_{i j}\right)$ of an $\mathrm{M} / \mathrm{M} / 1$ queue with service rate $c_{i j}$. As another example, if the links are wireless, $D_{i j}\left(f_{i j}\right)$ can measure the transmission power required for achieving rate $f_{i j}$ on the channel from $i$ to $j$. For example, if the link transmission rate $f_{i j}$ is determined by the transmission power $P_{i j}$ as $f_{i j}=W \log \left(1+K P_{i j}\right)$ for some constants $W, K>0,{ }^{4}$ then $P_{i j}=\frac{1}{K}\left(2^{f_{i j} / W}-1\right) \triangleq D_{i j}\left(f_{i j}\right)$, which is strictly increasing and convex in $f_{i j}$.

For analytical purposes, we further assume that $D_{i j}(\cdot)$ is continuously differentiable with derivative $d_{i j}(\cdot)$. From now on, we will more often refer to the derivatives $d_{i j}(\cdot)=D_{i j}^{\prime}(\cdot)$ as the (marginal) cost functions. By previous assumptions, $d_{i j}(\cdot)$ is positive and strictly increasing. To simplify notation, we denote the cost function on relay $i$ 's outgoing link by $D_{i}(\cdot)$ and its marginal cost function by $d_{i}(\cdot)$. Let $\lambda_{i}(t) \triangleq d_{s i}(t)+$ $d_{i}(t)$ denote the marginal cost on path $(s, i, w)$. The socially optimal traffic allocation is the one that minimizes the network cost $\sum_{(i, j)} D_{i j}\left(f_{i j}\right)$. Because link costs are strictly convex, the socially optimal allocation is uniquely characterized by

$$
\lambda_{i}\left(r_{i}^{*}\right)=\min _{j \in \mathcal{I}} \lambda_{j}\left(r_{j}^{*}\right)
$$

if $r_{i}^{*}>0$. That is, every path from $s$ to $w$ with positive flow has the minimum marginal cost among all paths. For otherwise, one can reduce the total cost by shifting an infinitesimal amount of flow from a path with non-minimum marginal cost to one with the minimum marginal cost.

We model the source $s$ and relays as selfish agents who must pay for the costs on their outgoing links. While $s$ has to send all its traffic out, it strives to do this with the minimum cost. On the other hand, a relay has an incentive to forward traffic for $s$ only if it is adequately rewarded in the form of payments. The amount of payment is determined as follows.

Each relay $i$ announces a charging function $P_{i}(\cdot)$, with $P_{i}(0)=0$, which specifies the payment $P_{i}\left(f_{i}\right)$ it demands

\footnotetext{
${ }^{3}$ Since $D_{i j}(0)$ is the sunk cost on link $(i, j)$, its value is irrelevant to the strategic choice of the agents participating in the game.

${ }^{4}$ Assume that with proper time or frequency scheduling, transmission on different links are non-interfering.
} 
should $s$ forward traffic of rate $f_{i}$ to it. For analytical purposes, we assume that $P_{i}(t)$ is continuously differentiable with the derivative being the pricing function $p_{i}(t)$. Note that each $P_{i}(\cdot)$ provides $s$ a continuum of options, namely the rate-charge pairs $\left(f_{i}, P_{i}\left(f_{i}\right)\right)$. The destination $w$ does not charge relays for receiving traffic. Besides $P_{i}(\cdot)$, each relay $i$ also proposes to $s$ a traffic share $\gamma_{i} \in[0,1]$, whose use will be explained in a moment. After learning $\left(P_{i}(\cdot), \gamma_{i}\right)_{i \in \mathcal{I}}, s$ decides on the traffic allocation and makes payments to the relays accordingly.

\section{Pricing Game}

The pricing games starts with each relay announcing its bid. Then, the source $s$ allocates traffic to the relays in a way that minimizes its total payment. Upon receiving traffic and payments from $s$, each relay is required to forward as much traffic as it receives to the destination $w$. The payoff of each relay in this game is the profit it makes through servicing the traffic, i.e., the payment from $s$ minus the total cost on its outgoing link. In this section, we will define the oligopoly pricing game more formally.

1) Bidding Strategy: For convenience, we henceforth adopt the equivalent charging model where $s$ pays $B_{i}\left(f_{i}\right) \triangleq$ $D_{s i}\left(f_{i}\right)+P_{i}\left(f_{i}\right)$ to $i$ and $i$ pays the link cost $D_{s i}\left(f_{i}\right)$ in addition to $D_{i}\left(f_{i}\right)$. Later on, we will more often refer to $B_{i}(\cdot)$ as $i$ 's charging function. As implied by our assumptions on $D_{s i}(\cdot)$ and $P_{i}(\cdot), B_{i}(\cdot)$ is continuously differentiable and $B_{i}(0)=0$. Denote the space of functions with the above properties by $\mathcal{B}$. For convenience, we will often use the derivative $\beta_{i}(t) \triangleq B_{i}(t)^{\prime}=d_{s i}(t)+p_{i}(t)$ to characterize the charging function.

Besides $B_{i}(\cdot)$, the bid submitted by $i$ also includes a scalar $\gamma_{i} \in[0,1]$ which specifies $i$ 's proposed traffic share. When $s$ allocates traffic to minimize its total payment, it takes the relays' proposals $\left(\gamma_{i}\right)_{i \in \mathcal{I}}$ into consideration if there is a tie among multiple optimal allocations. We will specify the tiebreaking rule in a moment. To summarize, each relay $i$ bids $Q_{i} \triangleq\left(B_{i}(\cdot), \gamma_{i}\right) \in \mathcal{B} \times[0,1]$.

2) Selfish Traffic Allocation: Upon receiving the relays' bids, $s$ allocates its traffic in the most cost efficient way. We use routing variables $\phi_{i} \triangleq f_{i} / R_{s}, i \in \mathcal{I}$ [17] to characterize the traffic allocation. Clearly, the vector of routing variables $\phi=\left(\phi_{i}\right)_{i \in \mathcal{I}}$ is feasible if it is nonnegative and $\sum_{i \in \mathcal{I}} \phi_{i}=1$.

To minimize its total payment to the relays, $s$ always adopts a routing vector $\phi^{*}$ in the set

$$
\mathcal{A} \triangleq \underset{\phi \text { feasible }}{\arg \min } \sum_{i \in \mathcal{I}} B_{i}\left(R_{s} \phi_{i}\right) .
$$

Note that $\mathcal{A}$ is determined by $\left(B_{i}(\cdot)\right)_{i \in \mathcal{I}}$. It is always nonempty, since the set of feasible $\phi$ is compact and the charging functions $\left(B_{i}(\cdot)\right)_{i \in \mathcal{I}}$ are continuous. For some charging function profiles $\left(B_{i}(\cdot)\right), \mathcal{A}$ has only one element, in which case $\phi^{*}$ is uniquely determined. For others, $\mathcal{A}$ has more than one element, i.e., $s$ is indifferent among multiple (optimal) allocations. In such cases, the following tie-breaking rule specifies which allocation $s$ chooses as $\phi^{*}$.
Tie-Breaking Rule: Let $\bar{\gamma}$ be the normalized version of $\gamma \triangleq$ $\left(\gamma_{i}\right)_{i \in \mathcal{I}}$, i.e, $\bar{\gamma}_{i}=\gamma_{i} /\left(\sum_{j} \gamma_{j}\right)$. If all $\gamma_{j}=0$, let $\bar{\gamma}_{i}=1 /|\mathcal{I}|$ for every $i$. Whenever $\mathcal{A}$ has multiple elements, $s$ chooses the one closest to $\bar{\gamma}$. To be more specific, define

$$
\mathcal{A}^{*} \triangleq \underset{\phi \in \mathcal{A}}{\arg \min }\|\phi-\bar{\gamma}\|,
$$

where $\|\cdot\|$ denotes the Euclidean norm. Notice that $\mathcal{A}^{*}$ is jointly determined by $\left(B_{i}(\cdot)\right)$ and $\left(\gamma_{i}\right)$. It is easy to see that $\mathcal{A}^{*}$ is non-empty. If $\mathcal{A}^{*}$ is a singleton, as typically is the case, let $\phi^{*}$ be that only element. Otherwise, $s$ chooses $\phi^{*}$ to be the allocation in $\mathcal{A}^{*}$ with the highest rank according to a predetermined lexicographic order. ${ }^{5}$ For example, $s$ can index the relays by $1,2, \cdots,|\mathcal{I}|$. An allocation $\phi$ is said to be lexicographically higher than $\phi^{\prime}$ if on the first coordinate $j$ where $\phi$ and $\phi^{\prime}$ differ, $\phi_{j}>\phi_{j}^{\prime}$.

With the above tie-breaking rule, we can write the traffic allocation $\phi^{*}$ as a function $\Phi$ of the relays' bids, i.e.,

$$
\phi^{*}=\left(\phi_{i}^{*}\right)_{i \in \mathcal{I}}=\Phi\left(\left(B_{i}(\cdot), \gamma_{i}\right)_{i \in \mathcal{I}}\right) .
$$

The traffic sent from $s$ to $i$ thus is $f_{i}=R_{s} \phi_{i}^{*}$.

Remark: The tie-breaking rule is intended to yield a definitive traffic allocation even when $s$ is indifferent among multiple (optimal) allocations. The total payment by $s$ remains the same irrespective of how the tie is resolved.

3) Payoff Function: The objective of selfish relays in forwarding traffic is to maximize their individual profits. Thus, we define the payoff function of a relay to be its profit as a function of its own as well as other relays' bids. Let $Q_{-i}$ denote the bids made by all relays other than $i$. The payoff function of relay $i$ is given by

$$
\begin{aligned}
\Gamma_{i}\left(Q_{i}, Q_{-i}\right) & \triangleq B_{i}\left(f_{i}\right)-\left[D_{s i}\left(f_{i}\right)+D_{i}\left(f_{i}\right)\right] \\
& =B_{i}\left(R_{s} \phi_{i}^{*}\right)-\left[D_{s i}\left(R_{s} \phi_{i}^{*}\right)+D_{i}\left(R_{s} \phi_{i}^{*}\right)\right] .
\end{aligned}
$$

On the RHS of (1), $f_{i}$ is the traffic going through links $(s, i)$, $(i, w)$ induced by the bids $Q_{i}, Q_{-i}$. Thus, the first term $B_{i}\left(f_{i}\right)$ represents the revenue $i$ earns from $s .{ }^{6}$ The summation in the bracket is the total cost incurred to $i$ for forwarding traffic $f_{i}$. In (2), $f_{i}$ is expressed in terms of $R_{s}$ and $\phi_{i}^{*}$. Recall that the routing decision by $s$ is determined as

$$
\phi^{*}=\Phi\left(\left(B_{j}(\cdot), \gamma_{j}\right)_{j \in \mathcal{I}}\right)=\Phi\left(Q_{i}, Q_{-i}\right) .
$$

Hence, $\phi^{*}$ is subject to change as $Q_{i}$ is varied.

4) Static Pricing Game - Formal Definition: We now formally define the (static) oligopoly pricing game (PG) as having the following components:

- The set of players $\mathcal{I}=\{$ relays in $\mathcal{G}\}=\mathcal{N} \backslash\{s, w\}$.

- Strategy of player $i: Q_{i}=\left(B_{i}(\cdot), \gamma_{i}\right) \in \mathcal{B} \times[0,1]$.

- Payoff to player $i$ : the profit made by servicing traffic $f_{i}$ :

$$
\Gamma_{i}\left(Q_{i}, Q_{-i}\right)=B_{i}\left(f_{i}\right)-\left[D_{s i}\left(f_{i}\right)+D_{i}\left(f_{i}\right)\right]
$$

\footnotetext{
${ }^{5}$ The lexicographic rule is intended to resolve any remaining ambiguity after utilizing the proposed shares $\left(\gamma_{i}\right)_{i \in \mathcal{I}}$. Its introduction is mainly for the purpose of being absolutely rigorous. It is never invoked at any equilibrium to be studied in the rest of the work.

${ }^{6}$ Recall that $i$ receives payment $B_{i}\left(f_{i}\right)$ from $s$ and pays $D_{s i}\left(f_{i}\right)$ by itself.
} 


$$
=\int_{0}^{f_{i}} \beta_{i}(r)-\lambda_{i}(r) d r
$$

where $\left(f_{i}\right)_{i \in \mathcal{I}}$ is the selfish traffic allocation induced by $\left\{Q_{i}, i \in \mathcal{I}\right\}$.

An oligopoly PG is fully characterized by $\left(R_{s},\left(\lambda_{i}(\cdot)\right)_{i \in \mathcal{I}}\right)$. Next, we will study the outcome of such games. In particular, we investigate whether a PG has an equilibrium, where no relay can increase its profit by unilaterally changing its bid, and when an equilibrium exists, how the resulting selfish traffic allocation compares to the socially optimal one.

\section{Best Response and Equilibrium}

Next, we formally define equilibria of an oligopoly pricing game. We start with the definition of the best response.

Definition 1: The best response set of $i$ given $Q_{-i}$ is

$$
\mathcal{B}_{i}\left(Q_{-i}\right)=\underset{Q_{i} \in \mathcal{B} \times[0,1]}{\arg \max } \Gamma_{i}\left(Q_{i}, Q_{-i}\right) .
$$

Definition 2: A bid profile $\left(Q_{i}\right)_{i \in \mathcal{I}}$ constitutes a (pure strategy) Nash equilibrium if for any $i \in \mathcal{I}, Q_{i} \in \mathcal{B}_{i}\left(Q_{-i}\right)$.

Definition 3: An equilibrium $\left(Q_{i}\right)_{i \in \mathcal{I}}$ is efficient if it induces the socially optimal traffic allocation. In this case, $\left(Q_{i}\right)_{i \in \mathcal{I}}$ is said to induce the social optimum.

In the next section, we will prove the existence of equilibria and analyze their efficiency in oligopoly pricing games.

\section{EQUILIBRIUM ANALYSIS}

\section{A. Best Response Conditions and Existence of Equilibria}

We first present the necessary and sufficient conditions for $Q_{i}$ to be relay $i$ 's best response to $Q_{-i}$. Define

$$
\begin{aligned}
B_{\hat{i}}(t) & \triangleq \min _{\sum_{j \in \mathcal{I} \backslash\{i\}} f_{j}=t} \sum_{j \in \mathcal{I} \backslash\{i\}} B_{j}\left(f_{j}\right) \\
& =\min _{\sum_{j \in \mathcal{I} \backslash\{i\}} f_{j}=t} \sum_{j \in \mathcal{I} \backslash\{i\}} \int_{0}^{f_{j}} \beta_{j}(r) d r .
\end{aligned}
$$

which gives the minimum payment $s$ has to make for transmitting traffic of rate $t$ through relays other than $i$. It is easy to show that $B_{\hat{i}}(t)$ is continuous and increasing. Its derivative, denoted by $\beta_{\hat{i}}(t)$, is in general piecewise continuous. For $t \in\left(0, R_{s}\right)$, let the left and right limits of $\beta_{\hat{i}}(\cdot)$ at $t$ be denoted by $\beta_{\hat{i}}(t)^{-}$and $\beta_{\hat{i}}(t)^{+}$.

Lemma 1: For any $i \in \mathcal{I}$ and any $Q_{-i}, Q_{i} \in \mathcal{B}_{i}\left(Q_{-i}\right)$ if and only if

(i)

$$
B_{i}(t) \geq B_{\hat{i}}\left(R_{s}\right)-B_{\hat{i}}\left(R_{s}-t\right)
$$

for all $t \in\left[0, R_{s}\right]$;

(ii)

$$
B_{i}\left(f_{i}^{*}\right)=B_{\hat{i}}\left(R_{s}\right)-B_{\hat{i}}\left(R_{s}-f_{i}^{*}\right),
$$

${ }^{7}$ It is understood that $\beta_{\hat{i}}(0)$ has only a right limit and that $\beta_{\hat{i}}\left(R_{S}\right)$ has only a left limit. where $f_{i}^{*}$ maximizes

$$
\bar{\Gamma}_{i}\left(f_{i} ; Q_{-i}\right) \triangleq B_{\hat{i}}\left(R_{s}\right)-B_{\hat{i}}\left(R_{s}-f_{i}\right)-D_{s i}\left(f_{i}\right)-D_{i}\left(f_{i}\right)
$$

over $f_{i} \in\left[0, R_{s}\right]$;

(iii) $\left(\gamma_{i},\left(\gamma_{j}\right)_{j \in \mathcal{I} \backslash\{i\}}\right)$ induces the tie-breaking rule of $s$ to generate $\phi_{i}^{*}=f_{i}^{*} / R_{s}$.

Limited by the space, we provide only some intuitive explanations for the lemma. By definition (3), $B_{\hat{i}}(r)$ represents the minimum cost that $s$ can achieve by forwarding traffic of rate $r$ to relays other than $i$. It will become evident in the next proof that from $i$ 's viewpoint, the competition from all other $j \in \mathcal{I}$ can be aggregated into a virtual competitor $\hat{i}$ using charging function $B_{\hat{i}}(\cdot)$. Since $s$ needs to pay $B_{\hat{i}}\left(R_{s}\right)$ when routing exclusively to $\hat{i}$, its payment would be no more than $B_{\hat{i}}\left(R_{s}\right)$ should $s$ optimally route to both $i$ and $\hat{i}$. Thus, the maximum revenue $i$ could expect to have when forwarding $f_{i}$ is $B_{\hat{i}}\left(R_{s}\right)-B_{\hat{i}}\left(R_{s}-f_{i}\right)$. It is then easy to see that $\bar{\Gamma}_{i}\left(f_{i} ; Q_{-i}\right)$ represents $i$ 's maximum profit by winning $f_{i}$ from $s$, and $f_{i}^{*}$ is $i$ 's optimal "market share". Charging function $B_{i}(\cdot)$ and proposed share $\gamma_{i}$ which satisfy the conditions in Lemma 1 induce $s$ to allocate the ideal "market share" $f_{i}^{*}$ to $i$ and give $i$ the maximum profit. This is because (5) implies that allocating $f_{i}^{*}$ to $i$ and the rest to $\hat{i}$ yields the same cost to $s$ as allocating all the traffic to $\hat{i}$, while conditions (4) and (5) combined imply that no other allocation costs strictly less than the above two schemes. Therefore, optimal allocations of $s$ include, at least, $\left(f_{i}^{*}, R_{s}-f_{i}^{*}\right)$ and $\left(0, R_{s}\right)$, where the two components are the traffic allocated to $i$ and $\hat{i}$, respectively. Condition (iii) is necessary and sufficient to ensure that $s$ opts for the former allocation. Moreover, since $s$ ends up paying $B_{\hat{i}}\left(R_{s}\right), i$ has maximally realized its profit potential.

Note that while $B_{i}(\cdot)$ satisfying (i) and (ii) always exists (e.g. let $B_{i}(t)=B_{\hat{i}}\left(R_{s}\right)-B_{\hat{i}}\left(R_{s}-t\right)$ for $\left.t \in\left[0, R_{s}\right]\right), \gamma_{i}$ satisfying (iii) may or may not exist. Thus, $\mathcal{B}_{i}\left(Q_{-i}\right)$ may be empty for some $Q_{-i}$. Nevertheless, we will show that (pure strategy) Nash equilibria always exist.

To better understand and visualize conditions (i) and (ii) in Lemma 1, we rewrite them succinctly in an integral form:

$$
\int_{0}^{t} \beta_{i}(r) d r \begin{cases}\geq \int_{0}^{t} \beta_{\hat{i}}\left(R_{s}-r\right) d r, & 0 \leq t \leq R_{s} \\ =\int_{0}^{t} \beta_{\hat{i}}\left(R_{s}-r\right) d r, & t=f_{i}^{*},\end{cases}
$$

where $f_{i}^{*}$ maximizes

$$
\bar{\Gamma}_{i}\left(f_{i}, Q_{-i}\right)=\int_{0}^{f_{i}} \beta_{\hat{i}}\left(R_{s}-r\right)-\lambda_{i}(r) d r,
$$

over $f_{i} \in\left[0, R_{s}\right]$. To gain an intuitive idea of the conditions, suppose $\beta_{\hat{i}}\left(R_{s}-r\right)$ and $\lambda_{i}(r)$ are given by the dashed and solid curves in Figure 2. A typical best response $\beta_{i}(r)$ is shown as the dotted curve.

\section{B. Efficient Equilibria}

We now analyze the allocation established by the oligopoly PG. Recall that given $\left(\beta_{i}(\cdot)\right)_{i \in \mathcal{I}}, s$ adopts the most cost efficient allocation $\left(f_{i}^{*}\right)_{i \in \mathcal{I}}$, which satisfies

$$
\beta_{i}\left(f_{i}^{*}\right)=\min _{j \in \mathcal{I}} \beta_{j}\left(f_{j}^{*}\right)
$$




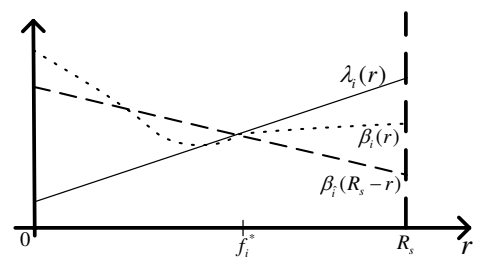

Fig. 2. Typical best response curve in an oligopoly.

whenever $f_{i}^{*}>0$. Should there be multiple optimal allocations, $s$ invokes the tie-breaking rule based on $\left(\gamma_{i}\right)_{i \in \mathcal{I}}$. Whether $\left(f_{i}^{*}\right)$ is equal to the optimal allocation $\left(r_{i}^{*}\right)$ depends on how $\left(\beta_{i}(\cdot), \gamma_{i}\right)$ are chosen by individual relays.

Theorem 1: The socially optimal traffic allocation of an oligopoly can always be induced by an equilibrium. That is, the price of stability of an oligopoly pricing game is one.

Proof: We prove the theorem by constructing an equilibrium that induces the socially optimal allocation $\left(r_{i}^{*}\right)$. Define $\lambda^{*} \triangleq \min _{j \in \mathcal{I}} \lambda_{j}\left(r_{j}^{*}\right)$. Let $\beta_{i}(r) \equiv \lambda^{*}$ and $\gamma_{i}=r_{i}^{*} / R_{s}$ for all $i$. Then, $\beta_{i}(r)=\beta_{\hat{i}}\left(R_{s}-r\right)=\lambda^{*}$ and condition (6) is satisfied for all $i$ with $f_{i}^{*}=r_{i}^{*}$. Since all the relays adopt the same constant pricing function, $s$ is indifferent among all feasible allocations. However, $\left(\gamma_{i}\right)_{i \in \mathcal{I}}$ induces $s$ to allocate $r_{i}^{*}$, which is equal to $i$ 's optimal traffic $f_{i}^{*}$, to each relay $i$. Therefore, $\left(\beta_{i}(\cdot), \gamma_{i}\right)$ constitutes an equilibrium which results in the allocation $\left(r_{i}^{*}\right)$.

Because the socially optimal allocation always exists, we can conclude that there always exists an efficient equilibrium for any oligopoly pricing game.

Although we used constant $\left(\beta_{i}(\cdot)\right)$ (or linear charging functions $\left.{ }^{8}\left(B_{i}(\cdot)\right)\right)$ to construct an efficient equilibrium in the proof of Theorem 1, efficient equilibria can be established by nonlinear charging functions as well. For instance, Figure 3 depicts an equilibrium in a duopoly PG where the two relays adopt $\beta_{1}(\cdot), \beta_{2}(\cdot)$ of a more general shape with $\gamma_{1}=r_{1}^{*} / R_{s}$ and $\gamma_{2}=\left(R_{s}-r_{1}^{*}\right) / R_{s}$. Notice that in a duopoly, $\beta_{\hat{1}}(t)=$ $\beta_{2}(t)$ and $\beta_{\hat{2}}(t)=\beta_{1}(t)$. Thus, $r_{1}^{*}$ is the optimal market share to 1 while $R_{s}-r_{1}^{*}$ is optimal to 2 . Although $s$ is indifferent among all feasible allocations, it follows the proposed shares $\left(\gamma_{1}, \gamma_{2}\right)$ so that the two relays each get its optimal share.

To derive a simple criterion for checking the efficiency of an equilibrium, we need to make the following distinction. A traffic allocation $\left(f_{i}\right)_{i \in \mathcal{I}}$ is monopolistic if $f_{m}=R_{s}$ for some relay $m$ and $f_{j}=0$ for all $j \neq m$. In this case, $m$ is called the dominant relay. An equilibrium is monopolistic if it induces a monopolistic allocation. An allocation is said to be competitive if there are at least two relays $i, j$ such that $f_{i}>0, f_{j}>0$. Such $i$ and $j$ are called competitive relays. An equilibrium is competitive if it induces a competitive allocation.

\footnotetext{
${ }^{8}$ Note that $P_{i}(\cdot)=B_{i}(\cdot)-D_{s i}(\cdot)$ generally is nonlinear when $B_{i}(\cdot)$ is linear.
}

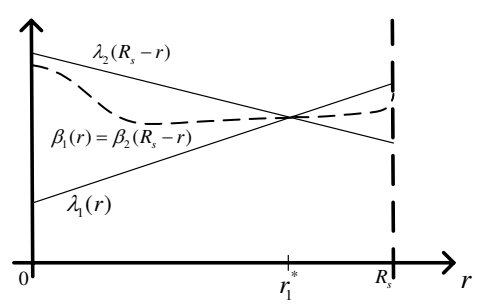

Fig. 3. General (focal) equilibrium in duopoly.

Theorem 2: If an oligopoly equilibrium is competitive, it must be efficient.

The proof, which is skipped here, makes use of a fundamental characteristic of a competitive equilibrium. That is, all the competitive relays set their equilibrium prices $\beta_{m}\left(f_{m}^{*}\right)$ equal to their marginal costs $\lambda_{m}\left(f_{m}^{*}\right)$; moreover their marginal costs are equal to each other. This is reminiscent of the classic Bertrand oligopoly competition. The Bertrand competition involves multiple producers with constant and identical unit costs who each declare a unit price to the market represented by a demand function. It is found that the only equilibrium of the Bertrand competition is one where all producers set their price equal to the cost. The equilibria of our oligopoly game have the same feature in terms of the prices and marginal costs evaluated at the equilibrium allocation point.

\section{Inefficient Equilibria}

Theorem 2 does not rule out the possibility of inefficient equilibria. In fact, an equilibrium may be inefficient if it is monopolistic. Essentially, a monopolistic equilibrium takes hold when a relay bids others out of competition to acquire the entire traffic. We call such behavior of the dominant relay manipulative pricing. The capability of manipulative pricing, however, stems from the relatively superior cost function of the dominant relay, as formalized in the next theorem. We skip the proof due to space limit.

Theorem 3: If an oligopoly equilibrium is monopolistic with dominant relay $m$, we must have

$$
\int_{0}^{R_{s}} \lambda_{m}(r) d r \leq \int_{0}^{R_{s}} \lambda_{j}(r) d r
$$

for any other relay $j$.

The next conclusion easily follows from Theorem 3 .

Corollary 1: If the socially optimal allocation of an oligopoly is monopolistic, then every equilibrium of the oligopoly is monopolistic and efficient.

It is shown next that there always exists a monopolistic equilibrium. Thus, we have the following conclusion.

Corollary 2: If the socially optimal routing of an oligopoly is competitive, then there exists an inefficient (monopolistic) equilibrium. 
Proof: We need only to show that there exists a monopolistic equilibrium in such an oligopoly. Let all $\beta_{i}(\cdot)$ be the same strictly decreasing function $\beta(\cdot)$ such that $\int_{0}^{t} \beta\left(R_{s}-r\right) d r \leq \int_{0}^{t} \lambda_{i}(r) d r$ for all $t \in\left[0, R_{s}\right)$ and $i \in \mathcal{I}$ but $\int_{0}^{R_{s}} \beta\left(R_{s}-r\right) d r=\int_{0}^{R_{s}} \lambda_{m}(r) d r$ where $m \in \arg \min _{i} \int_{0}^{R_{s}} \lambda_{i}(r) d r$. Also let $\gamma_{m}=1$ and $\gamma_{i}=0$ for all relays but $m$. Since $\beta(\cdot)$ is strictly decreasing, $\beta_{\hat{i}}(r)=\beta(r)$ for all $i$. By construction, $f_{i}^{*}=0$ is an ideal flow to $i \neq m$ (cf. (7)) and (6) holds with $f_{i}^{*}=0$ because $\beta_{i}(\cdot)=\beta_{\hat{i}}(\cdot)=\beta(\cdot)$ is strictly decreasing. From $m$ 's perspective, $\beta_{m}(\cdot)=\beta(\cdot)$ and $f_{m}^{*}=R_{s}$ jointly satisfy $m$ 's best response conditions (6)-(7). The proposed shares $\left(\gamma_{i}\right)$ guarantee each relay getting its proposed share even if $s$ is indifferent. So the monopolistic equilibrium is established.

\section{Price of Anarchy}

The price of anarchy, as a measure of loss of social efficiency due to selfish behavior of individual agents, was studied in the literature on selfish routing [7], [8]. In this work, the price of anarchy of an oligopoly PG is defined as follows.

Definition 4: The price of anarchy $\rho\left(R_{s},\left(\lambda_{i}(\cdot)\right)_{i \in \mathcal{I}}\right)$ of an oligopoly PG $\left(R_{s},\left(\lambda_{i}(\cdot)\right)_{i \in \mathcal{I}}\right)$ is the ratio of the maximum cost at an equilibrium to the socially optimal cost, i.e.,

$$
\rho\left(R_{s},\left(\lambda_{i}(\cdot)\right)_{i \in \mathcal{I}}\right) \triangleq \frac{\max _{\left(f_{i}\right) \in \mathcal{F} E} \sum_{i \in \mathcal{I}} \int_{0}^{f_{i}} \lambda_{i}(r) d r}{\min _{\left(f_{i}\right) \in \mathcal{F}} \sum_{i \in \mathcal{I}} \int_{0}^{f_{i}} \lambda_{i}(r) d r},
$$

where $\mathcal{F}^{E}$ is the collection of all the allocations that can be induced by an equilibrium of $\left(R_{s},\left(\lambda_{i}(\cdot)\right)_{i \in \mathcal{I}}\right)$ and $\mathcal{F}$ is the set of all feasible allocations.

The price of anarchy of an oligopoly PG is upper bounded by $|\mathcal{I}|$, the number of relays, when the marginal cost functions $\left(\lambda_{i}(\cdot)\right)$ are chosen from the class of nonnegative, strictly increasing and concave functions. However, the price of anarchy can be arbitrarily large when $\left(\lambda_{i}(\cdot)\right)$ are chosen from the class of nonnegative, strictly increasing and convex functions.

Theorem 4: If the cost derivatives $\left(\lambda_{i}(\cdot)\right)$ are nonnegative, strictly increasing and concave, $\rho\left(R_{s},\left(\lambda_{i}(\cdot)\right)\right)$ of an oligopoly pricing game is upper bounded by the number of relays $|\mathcal{I}|$. The upper bound is achieved when the marginal costs are identical and linear.

Unlike the selfish routing games studied in [7], [8], where the price of anarchy is independent of the topology [15], Theorem 4 indicates that $\rho\left(R_{s},\left(\lambda_{i}(\cdot)\right)_{i \in \mathcal{I}}\right)$ of an oligopoly PG explicitly depends on topology through $|\mathcal{I}|$. It implies that the more intensive (larger $|\mathcal{I}|$ ) the competition is, the more inefficient the market becomes if it is monopolized. The situation is even worse if $\left(\lambda_{i}(\cdot)\right)$ are convex .

Theorem 5: For a fixed number $|\mathcal{I}| \geq 2$ of relays and for any $M>0$, there exists an oligopoly $\left(R_{s},\left(\lambda_{i}(\cdot)\right)_{i \in \mathcal{I}}\right)$ with convex $\left(\lambda_{i}(\cdot)\right)$ such that $\rho\left(R_{s},\left(\lambda_{i}(\cdot)\right)_{i \in \mathcal{I}}\right) \geq M$.

\section{CONCLUSION}

This work presented a game-theoretic analysis of price competition in unicast networks with a single layer of parallel relays. The introduction of possibly nonlinear charging functions to the game enabled us to develop a much richer set of results than if we allowed only constant unit prices. While the socially optimal traffic allocation can always be induced by an equilibrium, the game may have inefficient equilibria as well. Furthermore, the existence of competition turns out to be a two-sided coin. On the one side, any competitive equilibrium must be efficient. On the other side, the conclusion that the price of anarchy of an oligopoly where relays have concave marginal costs is equal to the number of relays seems to suggest that more intense competition only makes inefficient (monopolistic) equilibria even worse. Efficiency loss can be still worse when marginal cost functions are convex. We found that the price of anarchy in this case can be arbitrarily large.

\section{REFERENCES}

[1] A. Blanc, Y. Liu, and A. Vahdat, "Designing incentives for peer-to-peer routing," in Proceedings of IEEE INFOCOM 2005, vol. 1, Mar. 2005.

[2] J. Crowcroft, R. Gibbens, F. Kelly, and S. Östring, "Modelling incentives for collaboration in mobile ad hoc networks," Performance Evaluation, vol. 57, no. 4, pp. 427-439, 2004.

[3] P. Marbach and Y. Qiu, "Cooperation in wireless ad hoc networks: a market-based approach," IEEE/ACM Transactions on Networking, vol. 13, pp. 1325-1338, Dec. 2005.

[4] L. Buttyan and J.-P. Hubaux, Security and Cooperation in Wireless Networks. Cambridge University Press, 2007.

[5] F. Kelly, A. Maulloo, and D. Tan, "Rate control in communication networks: shadow prices, proportional fairness and stability," Journal of the Operational Research Society, vol. 49, 1998.

[6] R. Cole, Y. Dodis, and T. Roughgarden, "Pricing network edges for heterogeneous selfish users.," in Proceedings of the 35th Annual ACM Symposium on Theory of Computing, pp. 521-530, Jun. 2003.

[7] T. Roughgarden and E. Tardos, "How bad is selfish routing," Journal of the ACM, vol. 49, no. 2, pp. 236-259, 2002.

[8] T. Roughgarden, "Selfish routing with atomic players," in Proceedings of the sixteenth annual ACM-SIAM symposium on Discrete algorithms, pp. 1184-1185, 2005.

[9] J. Shu and P. Varaiya, "Pricing network services," in Proceedings of IEEE INFOCOM 2003, vol. 2, Mar. 2003.

[10] L. He and J. Walrand, "Pricing differentiated internet services," in Proceedings of IEEE INFOCOM 2005, vol. 1, Mar. 2005.

[11] T. Basar and R. Srikant, "Revenue-maximizing pricing and capacity expansion in a many-users regime," in Proceedings of IEEE INFOCOM 2002, vol. 1, 2002.

[12] L. He and J. Walrand, "Pricing and revenue sharing strategies for internet service providers," in Proceedings of IEEE INFOCOM 2005, vol. 1, Mar. 2005.

[13] S. Shakkottai and R. Srikant, "Economics of network pricing with multiple ISPs," in Proceedings of IEEE INFOCOM 2005, vol. 1, Mar. 2005.

[14] D. Acemoglu and A. Ozdaglar, "Competition in parallel-serial networks," IEEE Journal on Selected Areas in Communications, pp. 11801192, Aug 2007.

[15] T. Roughgarden, "The price of anarchy is independent of the network topology," Journal of Computer and System Sciences, vol. 67, no. 2, pp. 341-364, 2003.

[16] R. Wilson, Nonlinear Pricing. Oxford University Press Inc, USA, 1993.

[17] R. Gallager, "A minimum delay routing algorithm using distributed computation," IEEE Transactions on Communications, vol. 25, no. 1, pp. 73-85, 1977. 